Voix et Images

volxetimages

\title{
Une industrie québécoise : le « rejaquettage »
}

\section{Richard St-Germain}

Volume 15, numéro 2 (44), hiver 1990

Pratiques illicites

URI : https://id.erudit.org/iderudit/200837ar

DOI : https://doi.org/10.7202/200837ar

Aller au sommaire du numéro

\section{Éditeur(s)}

Université du Québec à Montréal

\section{ISSN}

0318-9201 (imprimé)

1705-933X (numérique)

Découvrir la revue

\section{Citer cet article}

St-Germain, R. (1990). Une industrie québécoise : le « rejaquettage ». Voix et Images, 15(2), 236-246. https://doi.org/10.7202/200837ar d'utilisation que vous pouvez consulter en ligne.

https://apropos.erudit.org/fr/usagers/politique-dutilisation/ 


\section{Une industrie québécoise: le "rejaquettage"*}

\section{par Richard St-Germain, Université du Québec à Montréal}

Depuis quelques années déjà, nous travaillons à l'établissement d'un répertoire bibliographique de la littérature de grande consommation au Québec (1940-1960). Les particularités et les problèmes rencontrés pour l'établissement d'un tel répertoire sont l'absence quasi totale de témoignages écrits (encore moins publiés) ou de documents, la dispersion des témoins de première ligne, le brouillage des mémoires, l'absence de dépôt légal et le caractère souvent illicite de certaines publications. Sans compter le fait que la seule façon de connaître l'existence de tels titres est de les avoir en main. Et, pour ramasser tous ces livres, il faut effectuer du travail sur le terrain: courir les librairies d'occasion, les marchés aux puces, les "ventes de garage" et quelquefois consulter des collections privées.

Nous avons dressé ici un inventaire des titres et des éditeurs ayant eu recours à un procédé que nous nommons le "rejaquettage». Plusieurs éditeurs en firent une pratique courante, surtout dans les années 50 . Certains l'utilisaient de façon sporadique, alternant un titre en rejaquettage et un titre imprimé ici ${ }^{1}$. D'autres s'en servaient systématiquement et tous les livres qu'ils diffusaient étaient tirés d'éditions françaises ou de titres déjà publiés par des éditeurs québécois.

Le procédé était somme toute assez simple. On achetait, venant de France ou de Belgique, des cargaisons complètes de petits romans populaires et on en arrachait carrément les couvertures pour y brocher à la place une jaquette (c'està-dire, selon le Robert, une chemise à caractère publicitaire protégeant la couverture d'un livre relié ou broché; sauf qu'ici les couvertures ont été arrachées) portant le nom de l'éditeur québécois.

Prenons l'exemple d'un roman diffusé par les Éditions Normandy dans la collection "Amour Amoroso». La page 1 de la jaquette comporte plusieurs éléments: le nom de l'éditeur, l'identification de la collection, la photo d'un couple qui s'embrasse (et qui sert de logo à la collection), la photo et le nom d'une vedette de cinéma de l'époque, le numéro dans la collection, le nom de l'auteur et enfin un titre: À l'ombre de la Tour. Les pages 2 et 3 sont vides. La page 4 est utilisée par l'éditeur québécois pour offrir à ses lecteurs la possibilité de commander des livres par la poste. Ensuite vient le livre en tant que tel avec la page de titre sur

\footnotetext{
* La rédaction de cet article et les recherches qui l'entourent ont été rendues possibles grâce à des subventions du Fonds pour la formation de chercheurs et l'aide à la recherche (FCAR) et du Conseil de recherches en sciences humaines du Canada: Études canadiennes, outils de recherche, et s'intègrent dans le cadre du Groupe de recherche en lecture (GREL) à l'UQAM.

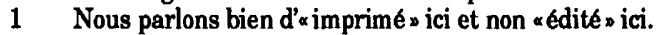


laquelle, en plus du nom de l'auteur, qui est le même que sur la page 1 de la jaquette, sauf que son prénom est écrit au complet, on remarque immédiatement que le titre n'est pas tout à fait le même: À l'ombre de la Tour Alix, et que l'éditeur est français: Bonne Presse. Maintenant, si l'on porte attention à la dernière page du livre, celle où sont inscrits normalement le nom de limprimeur et la date d'achevé d'imprimer, nous voyons bien que ce roman a été édité et imprimé par la Maison de la Bonne Presse, à Paris. Tout ce qu'il y a de québécois dans ce petit roman, c'est la jaquette et les lecteurs éventuels!

Cet exemple constitue l'une des formes que peut prendre le rejaquettage. Il arrivait parfois que la jaquette soit directement brochée sur la couverture d'origine ou que, sous la même jaquette portant un titre et un numéro de collection, on ne place pas toujours le même titre. Prenons l'exemple du numéro 10 de la collection "Éclair", série "Roman d'Amour": la jaquette porte le titre Un petit bonheur, sous lequel on trouve soit le roman la Vengeance de Lady Edwina, soit le roman Pâques de nos amours (2 romans sous un même titre)!

Le rejaquettage n'était donc pas la seule pratique "illicite» utilisée par les éditeurs. Il était également courant de changer le titre d'un roman. On recourait au " retitrage " surtout dans le cas de romans à tendance érotique et violente comme "Les aventures du Zodiaque", "Les aventures de Blankie et Blondie" et "Les aventures de la Môme Double-Shot ", tous publiés ici aux Éditions Paris Tour-Eiffel.

Ainsi, pour les "Aventures du Zodiaque", dont tous les titres sont de Gaston Martin, les titres français ne correspondent que très rarement à ceux de l'édition québécoise (tous les titres sont publiés en vertu d'une entente avec les éditions Neuilly 1951-1952-1953). Zodiaque, faut-il le savoir, est «LE HÉROS le plus sympathique de l'AVENTURE MODERNE".

Numéro et titre français

1 Et la mariée conduit le bal

5 On a volé une souris à Broadway

34 En plein turf

46 Il me la faut

70 Bon pour le compte

73 Au dessous de zéro

75 la Poupée aimait le Baroud

76 Faut toujours croire au Père Noël

83 Du plomb à revendre
Numéro et titre québécois

1 la Mariée a peur la nuit

5 On enlève les Pin-up à Broadway

34 l'Amour au clair de lune

43 Manque de pot

70 Bon pour l'amour

73 l'Amour au dessous de zéro

75 la Pépée aimait le Baroud

76 Faut toujours croire à l'amour

83 De l'amour à revendre

Et, pour en donner un second exemple, il en va de même de la collection "La Môme Double-Shot", dont l'auteur est Georges Maxwell, et qui était publiée en France aux Éditions le Condor, Paris, 1952-1953. La plupart des titres sont modifiés, d'une façon plus ou moins importante.

\section{Numéro et titre français}

1 Fallait pas me doubler

2 Va te faire voir!

3 Y manque une brique
Numéro et titre québécois

1 l'Ingénue scandale

2 Jeux de femmes

3 Fille d'amour 
4 la Belle se joue à deux

5 Sans bavure

6 Au premier de ces messieurs

7 C'est du gâteau

8 Quelques roses pour le tueur

9 Un pyjama de sapin

10 Rien ne va plus

11 C'ẹst ma tournée

12 les Squelettes ne jouent pas au pocker!

13 Pas de salades, j'en vends

14 Quel sale temps

15 Calibre 45... et culotte de soie

16 Du sang dans le champagne

17 J'peux pas l'encadrer
4 la Belle se joue à deux

5 Étrange Passion

6 Une belle garce

7 Trafic de filles

8 Boutons de roses... et d'autres

9 Avec et sans pyjama

10 Un mari qui a ses préférences

11 Drôles de caresses

12 Volupté

13 Prélude au désir

14 Pâmoison

15 Calibre 45... et culotte de soie

16 l'Amour au champagne

17 On cherche des femmes

Outre ces deux pratiques qu'on pourrait qualifier d'«illicites", il arrivait également que le titre d'un ouvrage soit conservé mais que le nom du romancier soit modifié. Par exemple, le roman français Maïté de Serge Laurac, pseudonyme de Pierre Genève, alors directeur des Éditions Eurédif sises au 8, rue de la Baume à Paris, était diffusé au Québec sous le titre Marie-Thérèse et signé Serge de la Baume.

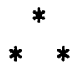

De telles pratiques d'éditions ne facilitent pas les recherches sur cette littérature de grande consommation. Qui correspond à qui ? Interrogation à laquelle il n'est guère facile de répondre sans connaître parfaitement personnages et lieux des actions. Pour peu que ceux-ci soient également changés, la seule solution est alors de posséder l'original et la (ou les) contrefaçon(s). Il reste encore bien des points à éclaircir et cela sera fait quand nous aurons réussi à mettre la main sur certains ouvrages manquants.

Il va de soi que la présente liste reste incomplète. Les titres ont été regroupés par maison d'édition; les descriptions comprennent le nom de la collection ou de la série dans laquelle les titres sont parus, le format des livres au demi-centimètre près, une brève description de la page 1 de la jaquette et la nationalité du ou des auteurs. Chaque fois que ce fut possible, la date et le nom de l'éditeur d'origine ont été indiqués. Lorsque le titre sur la jaquette et celui de la page de titre du livre ne correspondent pas, les deux sont indiqués et suivis, entre parenthèses, de l'endroit d'où ils sont tirés: (jaq.) pour la jaquette et (titre) pour la page de titre du livre.

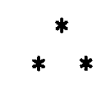

\section{LE CERCLE DU ROMAN POLICIER}

40 , rue Molière, Montréal

FORMAT: $11,5 \times 17 \mathrm{~cm}$ 
JAQUETTES: non illustrées, sauf un roman de Bertrand Vac ${ }^{2}$

AUTEURS: étrangers, sauf Bertrand Vac

Sans numérotation

Publiée dans les années 50, cette collection, qui ne semble compter que 29 titres, présentait des co-éditions et des rejaquettages des grandes collections françaises de l'époque. La couverture fade en deux couleurs (noir et couleur) présente le titre dans un cadre blanc. Seul l'Assassin dans l'hôpital (1956), le roman de Bertrand Vac (pseudonyme d'Aimé Pelletier), l'unique auteur québécois de la collection d'ailleurs, a une couverture illustrée. Le sigle est un homme à chapeau tenant un poignard dans la main droite. Sur les 29 titres, 12 sont des rejaquettages.

$\begin{array}{ll}\text { Date } & \text { Auteur } \\ 1955 & \text { Leslie Charteris } \\ 1955 & \text { Maurice B. Endrèbe } \\ & \\ 1955 & \text { Jacques Decrest } \\ 1955 & \text { Jacques Decrest } \\ 1955 & \text { Erle Stanley Gardner } \\ 1955 & \text { Patrick Quentin } \\ 1955 & \text { Edgar Box } \\ 1955 & \text { Andrew Garve } \\ 1955 & \text { Erle Stanley Gardner } \\ 1955 & \text { Rex Stout } \\ 1955 & \text { Rex Stout } \\ 1955 & \text { Erle Stanley Gardner }\end{array}$

Titre

le Saint à Rio (Paris, Fayard, 1955)

Gondoles pour le cimetière (Paris, Presses de la Cité, 1955)

les Complices de l'aube (Paris, Pierre Horey, 1955)

Dieu mesure le vent (Paris, Pierre Horey, 1955)

le Cadavre cavaleur (Paris, Presses de la Cité, 1955)

C'est ma faute (Paris, Presses de la Cité, 1955)

Feu d'enfer (Paris, Presses de la Cité, 1955)

Mort contre la montre (Paris, Presses de la Cité, 1955)

la Rousse se remue (Paris, Presses de la Cité, 1955)

la Montagne noire (Paris, Fayard, 1955)

Dans la plus stricte intimité (Paris, Fayard, 1955)

la Nymphe négligente (Paris, Presses de la Cité, 1955)

***

\section{COLLECTION ÉCLAIR}

SÉRIE: Roman d'amour

FORMAT: il differre selon le format de l'édition française

JAQUETTES: photos de vedettes de cinéma (identifiées)

AUTEURS: étrangers

Sans date

La numérotation est plutôt anarchique: une couverture avec un titre et un numéro peut contenir un autre titre. Il s'agit de très mauvais rejaquettages d'éditions françaises. On remarque que sous le titre Un petit bonheur, $\mathrm{n}^{0} 10$ de la collection, paraissent deux romans différents.
$N^{\circ} \quad$ Auteur
Titre
$7 \quad$ Non signé
Passeport français (titre)

2 Bertrand Vac n'est pas ce que l'on pourrait considérer un auteur de littérature populaire. Il a écrit plusieurs romans qui sont considérés comme classiques. 
8 Non signé

10 L. S. Junod

10 bis Steph

11 José Rioux

12 Pierre Peter

$18 \quad$ Non signé
Coeurs meurtris (jaq.)

(Paris, Montsouris, «Stella », 1953)

Marie-Jeanne et moi (titre)

Devant le désir (jaq.)

(Paris, Bayard, « Bonne Presse», 1956)

la Vengeance de Lady Edwina (titre)

Un petit bonheur (jaq.)

(Montréal, Paris Tour-Eiffel, «Amour», s.d.)

Pâques de nos amours (titre)

Un petit bonheur (jaq.)

(Montréal, France-Livre, «Mon Livre Favori», 10, s.d)

le Chardon d'argent (titre)

Pardon d'amour (jaq.)

(Paris, Montsouris, «Stella », 1953)

la Confession (titre)

Seul à aimer (jaq.)

(Paris, Férenczi, « Petit Livre», 1953)

le Coeur est maître (titre)

la Joie d'aimer (jaq.)

ÉDITIONS E. P. R. (?)

COLLECTION: Mon roman policier

SÉRIE: Jaune

FORMAT: $11 \times 15 \mathrm{~cm}$

JAQUETTES: photos de vedettes de cinéma (identifiées)

AUTEURS: étrangers

Sans date

Il s'agit de très mauvais rejaquettages d'éditions françaises. Les titres présentés sur les jaquettes ne correspondent pas à ceux des livres.

\begin{tabular}{|c|c|c|}
\hline$N^{0}$ & Auteur & Titre \\
\hline 4 & Andrew Wood & $\begin{array}{l}\text { l'Homme qui est revenu (titre) } \\
\text { le Juge des pendus (jaq.) } \\
\text { (Paris, Férenczi, « le Verrou *, 17, 1951) }\end{array}$ \\
\hline 6 & Jean Kery & $\begin{array}{l}\text { Seul contre la meute (titre) } \\
\text { le Rafle dans le bouge (jaq.) } \\
\text { (Paris, Férenczi, « le Verrou *, 16, 1951) }\end{array}$ \\
\hline 8 & Gustave Gailhard & $\begin{array}{l}\text { Dernière Mort de Marc Bigle (titre) } \\
\text { le Testament du Lord (jaq.) } \\
\text { (Paris, Férenczi, « le Verrou *, 10, 1950) }\end{array}$ \\
\hline
\end{tabular}

\section{ÉDITIONS NORMANDY}

167, Laurier Est, Montréal (même adresse que les éditions Paris Tbur-Eiffel et Du StLaurent?) 
SERIE: Zig Zag Police

FORMAT: $10,5 \times 16 \mathrm{~cm}$

JAQUETTES: photos. En médaillon en haut à gauche, une photo de Martine Carol, Brigitte Bardot ou Kim Novak. Au centre, une photo de vedettes de cinéma (identifiées) sauf celle du numéro 233 qui est illustrée.

AUTEURS: français

Sans date

Simples rejaquettages d'éditions françaises

\begin{tabular}{|c|c|c|}
\hline$N^{o}$ & Auteur & Titre \\
\hline 189 & J. A. Flanigham & $\begin{array}{l}\text { Sans un murmure } \\
\text { (Paris, De Lutèce, «Bill Disley *, 2, 1955) }\end{array}$ \\
\hline 190 & J. A. Flanigham & $\begin{array}{l}\text { Histoire d'avoir peur } \\
\text { (Paris, De Lutèce, «Bill Disley », 12, 1956) }\end{array}$ \\
\hline 191 & J. A. Flanigham & $\begin{array}{l}\text { Amour et mitraillette } \\
\text { (Paris, De Lutèce, « Bill Disley », 3, 1955) }\end{array}$ \\
\hline 192 & J. A. Flanigham & $\begin{array}{l}\text { Minutes d'angoisse } \\
\text { (Paris, De Lutèce, « Bill Disley «, 9, 1956) }\end{array}$ \\
\hline 193 & J. A. Flanigham & $\begin{array}{l}\text { Rien ne va plus } \\
\text { (Paris, De Lutèce, « Bill Disley », 10, 1956) }\end{array}$ \\
\hline 194 & J. A. Flanigham & $\begin{array}{l}\text { l'Étonnant Mister Black } \\
\text { (Paris, De Lutèce, «Bill Disley », 11, } 1956\end{array}$ \\
\hline 195 & J. A. Flanigham & $\begin{array}{l}\text { Du fil à retorde } \\
\text { (Paris, De Lutèce, « Bill Disley », 6, 1955) }\end{array}$ \\
\hline 196 & J. A. Flanigham & $\begin{array}{l}\text { Voluptés rares et morts subites } \\
\text { (Paris, De Lutèce, « Bill Disley *, 7, 1956) }\end{array}$ \\
\hline 199 & Marcel Allain & $\begin{array}{l}\text { Deux Blondes } \\
\text { (Paris, Férenczi, «le Verrou *, 12, 1951) }\end{array}$ \\
\hline 202 & Gustave Gailhard & $\begin{array}{l}\text { la Seconde Mort de Marc Bigle (titre) } \\
\text { Mort et seconde mort (jaq.) } \\
\text { (Paris, De Lutèce, « Bill Disley *, 6, 1950) }\end{array}$ \\
\hline 203 & Gille Hersay & $\begin{array}{l}\text { le Chant des suicidés } \\
\text { (Paris, Férenczi, «le Verrou *, 14, 1951) }\end{array}$ \\
\hline 208 & Gustave Gailhard & $\begin{array}{l}\text { la Police en alerte } \\
\text { (Paris, Férenczi, «le Verrou «, 27, 1951) }\end{array}$ \\
\hline 215 & Gille Hersay & $\begin{array}{l}\text { la Mort aux ongles longs } \\
\text { (Paris, De Lutèce, « Bill Disley », 7, 1950) }\end{array}$ \\
\hline 225 & Marcel Allain & $\begin{array}{l}\text { Ce n'est pas lui } \\
\text { (Paris, De Lutèce, «Bill Disley », 24, 1951) }\end{array}$ \\
\hline 233 & Patrick Vander & $\begin{array}{l}\text { On a pris les papiers du mort } \\
\text { (Paris, Férenczi, « Police et Mystère», 65, 1954) }\end{array}$ \\
\hline 306 & Harry Edmonds & $\begin{array}{l}\text { le Mystère de la côte est } \\
\text { (Paris, De Lutèce, «Bill Disley *, 33, 1951) }\end{array}$ \\
\hline
\end{tabular}

SÉRIE: Cupidon (romans d'amour) FORMAT: $10,5 \times 16 \mathrm{~cm}$ 
JAQUETTES: médaillon en haut à gauche: un petit Cupidon; photos centrales: vedettes de cinéma (souvent identifiées)

AUTEURS: étrangers

Sans date

Rejaquettages des Éditions Paris Tour-Eiffel et des Éditions France-Livre (éditeur québécois de certaines collections des Éditions Férenczi.)

\begin{tabular}{|c|c|}
\hline$N^{0}$ & Auteur \\
\hline 167 & Line Harvey \\
\hline 168 & Annie \& Pierre Hot \\
\hline 169 & Régine Perrault \\
\hline 170 & Francine Robert \\
\hline 171 & Magda Contino \\
\hline 172 & Maryl Constant \\
\hline 173 & L. S. Junod \\
\hline 174 & Marie-Reine Aghion \\
\hline 175 & Marie-Reine Aghion \\
\hline 176 & Rémi Vercel \\
\hline 177 & Noël Monier \\
\hline 178 & Marie-Reine Aghion \\
\hline 179 & Lise De Cere \\
\hline 180 & Magda Contino \\
\hline 181 & Colette Lambert \\
\hline 182 & Annie \& Pierre Hot \\
\hline 183 & Non signé \\
\hline 184 & Dory \\
\hline 185 & Non signé \\
\hline 186 & Non signé \\
\hline 187 & Steph \\
\hline
\end{tabular}

Titre

le Soleil du bonheur

(Montréal, PTFL, *Amour *, 33, s.d.)

l'Heure du destin

(Montréal, PTFL, *Amour *, s.d.)

Aimer encore

(Montréal, PTFL, *Amour *, s.d.)

Un parfum de jeunesse, un parfum d'amour

(Montréal, PTFL, *Amour *, 17, s.d.)

l'Amour s'enfuit

(Montréal, PTFL, *Amour *, s.d.)

le Choix de Marie-France

(Montréal, PTFL, *Amour *, 38, s.d.)

la Vengeance de Lady Edwina

(Montréal, PTFL, *Amour *, s.d.)

Lui et moi

(Montréal, PTFL, *Amour *, s.d.)

l'Amour? un masque

A la recherche du bonheur

(Montréal, PTFL, «Amour *, 27, s.d.)

l'Amour joue et gagne

(Montréal, PTFL, *Amour *, 43, s.d.)

la Châtelaine de Chasseloup

(Montréal, PTFL, « Amour *, s.d.)

le Château sans belle

l'Aventure brésilienne

(Montréal, PTFL, «Amour «, 57, s.d.)

la Maison du bonheur

(Montréal, PTFL, «Amour *, 24, s.d.)

la Boîte à bonheur

(Montréal, PTFL, «Amour «, 60, s.d.)

le Château des sept douleurs

le Héros de Jaqueline

(Paris, S. E. P. I. A., 1952)

le Destin vengeur

la Marque de la honte

Pâques de nos amours

(Paris, Férenczi, «Mon Livre Favori», 886, s.d.; au Canada: Montréal, France-Livre, *Mon Livre

Favori *, 10, s.d.) 
SÉRIE: Amour Amoroso

FORMAT: $10,5 \times 16 \mathrm{~cm}$ et $10 \times 14 \mathrm{~cm}$

JAQUETTES: médaillon en haut à gauche: un couple qui s'embrasse; photo centrale: vedettes de cinéma (souvent identifiées)

AUTEURS: étrangers

Sans date

Rejaquettages d'éditions françaises

\begin{tabular}{|c|c|c|}
\hline$N^{0}$ & Auteur & Titre \\
\hline 70 & Suzanne Carpentier & $\begin{array}{l}\text { les Forges mystérieuses } \\
\text { (Paris, Montsouris, «Stella », 1953) }\end{array}$ \\
\hline 75 & Edouard de Keyser & $\begin{array}{l}\text { Si elle savait } \\
\text { (Paris, Montsouris, «Stella «, 1953) }\end{array}$ \\
\hline 76 & Claude Véla & $\begin{array}{l}\text { la Princesse aux étoiles } \\
\text { (Paris, Montsouris, « Stella », 1953) }\end{array}$ \\
\hline 77 & Marie De Cinquy & $\begin{array}{l}\text { À l'ombre de la tour Alix } \\
\text { (Paris, Bayard, «la Bonne Presse», 1955) }\end{array}$ \\
\hline 80 & Netty Delance & $\begin{array}{l}\text { Esclave du désir } \\
\text { (Paris, Férenczi, « Petit Livre», 1710, 1953) }\end{array}$ \\
\hline 81 & Pierre Peter & $\begin{array}{l}\text { Et l'amour triomphe } \\
\text { (Paris, Férenczi, « Petit Livre , 1705, 1953) }\end{array}$ \\
\hline 82 & Hélène Marval & $\begin{array}{l}\text { la Femme enchaînée } \\
\text { (Paris, Férenczi, *Petit Livre», 1703, 1953) }\end{array}$ \\
\hline 83 & Janine Mariel & $\begin{array}{l}\text { l'Obstacle secret } \\
\text { (Paris, Férenczi, « Petit Livre», 1697, 1953) }\end{array}$ \\
\hline 92 & Pierre Korab & $\begin{array}{l}\text { Sous l'aile des pagodes } \\
\text { Paris, Montsouris, «Stella *, 1952) }\end{array}$ \\
\hline 93 & Léo Henri & $\begin{array}{l}\text { la Chimère de porcelaine (titre) } \\
\text { Talisman d'amour (jaq.) } \\
\text { (Paris, Montsouris, «Stella *, 1953) }\end{array}$ \\
\hline 94 & Paule Antoine & $\begin{array}{l}\text { les Cendres du passé } \\
\text { (Paris, Montsouris, «Stella *, 1953) }\end{array}$ \\
\hline 95 & José Rioux & $\begin{array}{l}\text { le Chardon ardent } \\
\text { (Paris, Montsouris, «Stella «, 1953) }\end{array}$ \\
\hline 119 & Anna Michel & $\begin{array}{l}\text { Notre amour est le plus fort } \\
\text { (Paris, Férenczi, « Petit Livre «, 1752, 1954) }\end{array}$ \\
\hline 126 & Alba & $\begin{array}{l}\text { Lendemain d'amour } \\
\text { (Paris, Férenczi, *Petit Livre }, 1750,1954 \text { ) }\end{array}$ \\
\hline 142 & Sylvaine & $\begin{array}{l}\text { le Tendre ennemi (titre) } \\
\text { Ma vie vous appartient (jaq.) } \\
\text { (Paris, Férenczi, «Petit Livre }, 1730,1953 \text { ) }\end{array}$ \\
\hline 143 & France Noël & $\begin{array}{l}\text { les Amours de Caroline (titre) } \\
\text { Carole amoureuse (jaq.) } \\
\text { (Paris, Férenczi, « Petit Livre }, 1732,1953 \text { ) }\end{array}$ \\
\hline
\end{tabular}


$\begin{array}{lll}n ? \quad \text { Jeanne Moreau-Jousseaud } & \begin{array}{l}\text { le Larron d'honneur (titre) } \\ \text { Né d'une heure de folie (jaq.) } \\ \text { (Paris, Férenczi, «Notre coeur *, 21, 1953) } \\ \text { le Lien d'amour } \\ \text { (Paris, Férenczi, «Notre cœur *, 22, 1953) }\end{array}\end{array}$

SÉRIE: Notre cœur

FORMAT: $10,5 \times 16 \mathrm{~cm}$

JAQUETTES: photos et illustrées

AUTEURS: étrangers

Sans date

Rejaquettages d'édition française

\begin{tabular}{|c|c|c|}
\hline$N^{0}$ & Auteur & Titre \\
\hline 24 & Claude Belval & $\begin{array}{l}\text { la Fiancée des sables } \\
\text { (Paris, Férenczi, «Notre cœur», 21, 1953) }\end{array}$ \\
\hline 25 & Mona Gloria & $\begin{array}{l}\text { la Guerre des cœurs } \\
\text { (Paris, Férenczi, « Notre cœur», 23, 1953) }\end{array}$ \\
\hline
\end{tabular}

SÉRIE: Larmes d'amour

FORMAT: $13 \times 17 \mathrm{~cm}$

JAQUETTES: photos de vedettes de cinéma (identifiées)

Auteurs : étrangers

Sans date

Rejaquettages de la collection «Roman complet" des Éditions Fayard

\begin{tabular}{|c|c|c|}
\hline$N^{0}$ & Auteur & Titre \\
\hline 501 & René Trotet de Bargis & Amour fleur merveilleuse \\
\hline 508 & René de Pont-Jest & Crimes d'un ange \\
\hline 514 & Charles Mérouvel & Un lys au ruisseau \\
\hline 515 & Guy Desvaux & Je crois à l'amour \\
\hline 517 & Jean Thino & $\begin{array}{l}\text { la Nymphe endormie (titr } \\
\text { Vers l'amour (jaq.) }\end{array}$ \\
\hline 520 & Jacques Chanteuges & Jours de folies \\
\hline 526 & Robert Navailles & $\begin{array}{l}\text { l'Amour en danger (titre) } \\
\text { Danger d'amour (jaq.) }\end{array}$ \\
\hline
\end{tabular}

SÉRIE: Amour ardent

FORMAT: $10,5 \times 16 \mathrm{~cm}$

JAQUETTES: photos

AUTEURS : étrangers

Sans date

Rejaquettage d'édition française 
$\begin{array}{ll}N^{\circ} & \text { Auteur } \\ 51 & \text { J. Des Gruets }\end{array}$

SÉRIE: Romans d'amour $\mathbf{Z}$

FORMAT: $10,5 \times 16 \mathrm{~cm}$

JAQUETTES: photos

AUTEURS: étrangers

Sans date

Rejaquettage d'édition française

$\begin{array}{ll}N^{\circ} & \text { Auteur } \\ 49 & \text { Andrée Vertiol }\end{array}$

Titre

l'Amour du Maréchal de Concini

(Paris, De Lutèce, «Cape et d'épée *, 1956)
Titre

le Reflet (titre)

Magie de l'amour (jaq.)

(Paris, Montsouris, «Stella *, 1952)

SÉRIE: Hors collection

FORMAT: $10,5 \times 16 \mathrm{~cm}$

JAQUETTES: photos

AUTEURS: étrangers

Sans date

Rejaquettage d'édition française

$\begin{array}{ll}N^{\circ} & \text { Auteur } \\ ? ? & \text { Roger Flouriot }\end{array}$

Titre

Cécilia (titre)

Amoureusement (jaq.)

(Paris, Montsouris, « Stella *, 1952)

***

\section{ÉDITIONS DU ST-LAURENT}

167, Laurier Est, Montréal (même adresse que Paris Tour-Eiffel et éditions Normandy)

SÉRIE: Police

FORMAT: $10,5 \times 16 \mathrm{~cm}$

JAQUETTES: la plupart sont illustrées

AUTEURS: étrangers

Sans date

Certains titres sont des rejaquettages des éditions françaises; les autres sont imprimés par l'Imprimerie nationale à Joliette.
$N^{\circ}$ Auteur
9 J. A. Flanigham
Titre
11 J.A. Flanigham
l'Étonnant Mister Black
(Paris, De Lutèce, « Bill Disley *, 11, 1956)
13 J. A. Flanigham
Histoire d'avoir peur
(Paris, De Lutèce, «Bill Disley », 12, 1956)
Fait comme un rat
(Paris, De Lutèce, «Bill Disley *, 13, 1956) 


\begin{tabular}{|c|c|c|}
\hline 16 & André Firmin & $\begin{array}{l}\text { Ces cadavres sont mes amies } \\
\text { (Paris, De Lutèce, * Noire et Rose }, 33,1956 \text { ) }\end{array}$ \\
\hline 18 & René Rennes & $\begin{array}{l}\text { le Chinois voulait mourir à } 5 \text { hres } \\
\text { (Paris, E. I. M., s.d.) }\end{array}$ \\
\hline 22 & Maurice Limat & $\begin{array}{l}\text { l'Assassin est mort deux fois } \\
\text { (Montréal, Paris Tour-Eiffel, * Police», 6, s.d.) }\end{array}$ \\
\hline חר & J. A. Flanigham & $\begin{array}{l}\text { Une histoire du tonnerre } \\
\text { (Paris, De Lutèce, «Bill Disley », 3, 1955) }\end{array}$ \\
\hline
\end{tabular}

SÉRIE: Tendresse

FORMAT: $10,5 \times 16 \mathrm{~cm}$

JAQUETTES: photos

AUTEURS: étrangers

Sans date

Un seul rejaquettage des Éditions Paris Tour-Eiffel.

$\begin{array}{cll}N^{\circ} & \text { Auteur } & \text { Titre } \\ 7 & \text { Jean d'Iberville } & \text { la St-Jean des amours } \\ & & \text { (Montréal, Paris Tour-Eiffel, «Amour *, s.d.) }\end{array}$

SÉRIE: Amour

FORMAT: $10,5 \times 16 \mathrm{~cm}$

JAQUETTES: photos

AUTEURS: étrangers

Sans date

La plupart des titres sont imprimés par l'Imprimerie nationale ou par l'Imprimerie Berthier. Les autres sont des rejaquettages des éditions françaises.

$\begin{array}{cll}N^{\circ} & \text { Auteur } & \text { Titre } \\ 6 & \text { R. Gauthier } & \begin{array}{l}\text { Cette nuit-là } \\ \text { (Paris, De Lutèce, «Noire et Rose», 1956) }\end{array} \\ 7 & \text { Claude Bergerye } & \begin{array}{l}\text { Premier Mensonge } \\ \text { (Paris, E. I. M., s.d.) } \\ \text { la Jeune Fille au bouquet } \\ \text { (Paris, E. I. M., s.d.) }\end{array} \\ 12 & \text { Claudine Lepage } & \begin{array}{l}\text { le Secret de madame Allory } \\ \text { (Paris, Nord, 1949) } \\ \text { Un magicien passa } \\ \text { (Paris, Parvillée, 1946) }\end{array} \\ 17 & \text { Annie \& Pierre Hot } & \begin{array}{l}\text { Brigitte se taira } \\ \text { (Paris, Parvillée, 1946) }\end{array} \\ 19 & \text { Jean Miroir } & \begin{array}{l}\text { À la rencontre de l'amour } \\ \text { (Montréal, Paris Tour-Eiffel, «Amour*, s.d.) }\end{array} \\ 20 & \text { Camille Marbo } & \end{array}$

